\title{
The Frontier of High Energy Physics and the Large Hadron Collider
}

\author{
Kalanand Mishra \\ Fermi National Accelerator Laboratory, Batavia, Illinois 60510, USA \\ kalanand@fnal.gov
}

\begin{abstract}
High Energy Physics explores the most fundamental questions about the nature of the universe, e.g., basic building blocks of matter and energy, existence of the smallest sub-atomic particles, dark matter, dark energy etc. The Large Hadron Collider (LHC) is the most powerful accelerator on earth located near Geneva, Switzerland. It recreates the conditions just after the Big Bang by colliding two proton beams head-on at very high energy every 25-50 nanosecond. With the recent discovery of Higgs boson, the LHC is firmly marching on to explore the $\mathrm{TeV}$ energy scale.
\end{abstract}

Keywords: High Energy Physics, Large Hadron Collider, LHC.

PACS: $12.15 . J i, 12.38 . \mathrm{Qk}, 13.85 . \mathrm{Rm}$

\section{INTRODUCTION}

Everything, including ourselves, is made of matter whose underlying structure is governed by basic laws of physics. The goal of high-energy particle physics is to understand the fundamental constituents of matter and their mutual interactions by looking at the subatomic particles that make up atoms.

\section{THE BUILDING BLOCKS OF THE UNIVERSE}

Most of us are familiar with the concept of atoms and understand that they are made up of protons, neutrons, and electrons. However, if we zoom in closer, we learn that of these well known particles, only the electron is "fundamental," meaning that is isn't composed of smaller particles as far as we know. Protons are composite particles, i.e., they are made up of even smaller particles. These particles are called "quarks" after a line from James Joyce's Finnegan's Wake ("Three Quarks for Muster Mark"). Like protons, neutrons are also made of quarks. There are 6 types of quarks that we have created and measured in laboratory: the up quark, the down quark, the charm quark, the strange quark, the top quark, and the bottom quark. A proton is two up quarks and a down quark, and a neutron is an up dark and two down quarks. The up quark has charge $+2 / 3$ and the down quark has charge $-1 / 3$, so we recover the well-known properties that a proton has charge +1 and a neutron has charge 0 . The quarks inside a proton or neutron feel strong nuclear force. The things that bind quarks together, that make them stick to each other, are appropriately known as gluons.

\section{WEAK FORCE: THE REASON WHY WE EXIST}

Processes such as neutron decay can only be explained by introducing yet another new force of nature. By nuclear standards, the 15-minute lifetime of the neutron is extremely long (typical lifetimes of subatomic particles are in the range of a pico-second or smaller!), indicating a very weak process at work. Accordingly, the new force of nature is called the "weak force" and was first discovered in the neutron decay (also referred to as "beta decay"). Despite its slow rate and short range, the weak force plays a crucial role in the make-up of the world we observe. The sun would not burn without it since the weak interaction causes the formation of deuterium, then two deuterium nuclei fuse into helium, which provides the fuel for the sun. In fact, the weak force is responsible for our existence. If weak force didn't exist, there would be no atoms, no molecules, and no stable matter. In short, the word would not exist.

The weak interaction is the only process in which a quark can change to another quark, or a lepton (electron or muon) to another lepton. This is possible 
because the weak interaction involves the exchange of messenger particles known as the $\mathrm{W}$ and the $\mathrm{Z}$ bosons, which are heavyweights among the elementary particles - almost 100 times as massive as the proton. The masses of these bosons are significant because they act as force carriers; their masses thus limit the range of the weak interaction. The electromagnetic force, by contrast, has an infinite range because its force carrier (the photon) is massless.

\section{THE HIGGS BOSON}

The generally accepted picture of particle physics is known as the Standard Model [1]. This collection of theories is the most successful physical model ever known and is able to make predictions that agree with experiment to unprecedented accuracies. The Higgs boson is a massive elementary particle predicted to exist by the Standard Model [2]. It plays a unique role in the Standard Model, by explaining why the other elementary particles are massive. In particular, the Higgs boson would explain why the photon has no mass, while the $\mathrm{W}$ and $\mathrm{Z}$ bosons are very heavy. Elementary particle masses, and the differences between electromagnetism (mediated by the photon) and the weak force (mediated by the W and $\mathrm{Z}$ bosons), are critical to many aspects of the structure of microscopic (and hence macroscopic) matter. However, until very recently no experiment had directly detected the existence of the Higgs boson. The CMS and ATLAS experiments at the Large Hadron Collider (LHC) at CERN in Geneva reported the first experimental evidence of the Higgs boson's existence on July 4, 2012. Subsequently, the Higgs boson mass has been measured to be about $125 \mathrm{GeV}$. The Higgs boson is often referred to as "the God particle" by the media since this is the particle that gives mass to every other particle.

\section{LARGE HADRON COLLIDER}

The Large Hadron Collider (LHC) is the world's largest and highest-energy particle accelerator intended to collide two proton beams at an energy of $14 \mathrm{TeV}$ (currently running at $8 \mathrm{TeV}$ ) $[3,4]$. It lies in a tunnel 17 miles in circumference at the Franco-Swiss border near Geneva, Switzerland. The Large Hadron Collider was built by the European Particle Physics Laboratory (CERN) in collaboration with over 10,000 scientists and engineers from over 50 countries as well as hundreds of universities and laboratories.

On 17 December 2012 the first three-year LHC proton run came to a successful end. This remarkable run accomplished several performance milestones. The luminosity, a crucial parameter measuring the rate of collisions of an accelerator, reached a value of $7.7 \times 10^{33} \mathrm{~cm}^{-2} \mathrm{~s}^{-1}$, the highest instantaneous luminosity ever achieved at any hadron collider.

In the beginning of 2013 (January-February), the LHC collided protons with lead ions before going into a long maintenance stop until the end of 2014. Running will resume in 2015 with increased collision energy of $13 \mathrm{TeV}$ and another increase in luminosity.

\section{THE LHC EXPERIMENTS}

ATLAS and CMS are general-purpose particle physics experiments $[5,6]$. Designed to see a wide range of particles and phenomena produced in LHC collisions, each involves approximately 2,000 physicists from some 35 countries [4]. Having two independently designed detectors is vital for crossconfirmation of any new discoveries made. For example, the two experiments independently discovered a Higgs boson of mass $125 \mathrm{GeV}$ and then jointly made the announcement on July 4, 2012. Higgs production is one of the most demanding hypothesized processes in terms of required detector resolution and background discrimination. ATLAS and CMS feature full coverage, $4 \pi$-detectors to measure precisely the energies, directions and identity of all the particles produced in proton-proton collisions. Realizing this goal has required the collaborative efforts of enormous teams of people from around the world.

\section{OUTLOOK}

The LHC was built to answer several key scientific questions of the $21^{\text {st }}$ century. Why elementary particles have mass? How they acquire mass? What mechanism is responsible for mass generation? Why is gravity so many orders of magnitude weaker than the other three fundamental forces? Is Supersymmetry realized in nature, implying that for each known particle there is an extra particle to be discovered? Why are there apparent violations of the symmetry between matter and antimatter? What is the nature of dark matter and dark energy? Are there extra dimensions of space, and can we detect them?

With the discovery of a Higgs-like boson of mass about $125 \mathrm{GeV}$ at the $\mathrm{LHC}$, we have entered a new era of particle physics. For the first time in 50 years we now have at least a partial answer to the question regarding the mechanism responsible for mass generation. All other questions are still mostly open. Several years of LHC running is needed to accumulate enough data to potentially answer them. In the coming years at the LHC, we will measure Higgs boson interaction at the level of $10 \%$ precision or better and will probe physics beyond the SM at multi-TeV scale. 


\section{ACKNOWLEDGMENTS}

I would like to thank the local organizers of the Padjadjaran International Physics Symposium 2013 for their warm hospitality. Fermilab is operated by Fermi Research Alliance, LLC under Contract No. DEAC02-07CH11359 with the United States Department of Energy.

\section{REFERENCES}

1. Steven Weinberg, The First Three Minutes: A Modern View of the Origin of the Universe, $2^{\text {nd }}$ ed., Basic Books, New York (1993).

2. G. Kane, J. F. Gunion, S. Dawson and H. E. Haber, The Higgs Hunter's Guide, Westview Press, Boulder, Colorado (2000).

3. Lyndon Evans, The Large Hadron Collider, EFPL Press, Laussanne, Switzerland (2009).

4. Dan Green, At the Leading Edge: The ATLAS and CMS LHC Experiments, World Scientific Publishing Company, Singapore (2011).

5. S. Chatrchyan et al. (CMS Collaboration), "The CMS Experiment at the CERN LHC", JINST 3, S08004 (2008).

6. G. Aad et al. (ATLAS Collaboration), "The ATLAS Experiment at the CERN Large Hadron Collider", JINST 3, S08003 (2008). 